\title{
Der Kenntnisstand der Dermatologen in Praxis und Klinik zum Melanom
}

\author{
TED-Befragungen während eines Satelliten-Symposiums \\ der DDG-Tagung in Berlin
}

\author{
R. Dummer ${ }^{1}$ \\ J. Ulrich ${ }^{2}$ \\ S. Lischner ${ }^{3}$ \\ M. Volkenandt ${ }^{4}$ \\ C. Garbe ${ }^{5}$ \\ A. Hauschild ${ }^{3}$
}

\section{The Knowledge Concerning the Management of Melanoma of Dermatologists \\ in Private Practice and in Hospitals}

\section{Zusammenfassung}

Die Diskussion um die optimale Versorgung von Patienten mit Melanom der Haut wird kontrovers geführt. Mittels TED wurden zu Schlüsselfragen der Versorgung von Melanompatienten den Teilnehmern eines Symposiums fünf Multiple-Choice-Fragen gestellt und ausgewertet. Dies geschah vor und nach einem Vortrag zu diesem Thema. Somit konnte der Einfluss des Vortragenden auf das Meinungsbild des Auditoriums überprüft werden.

\section{Einleitung}

Während der 41. Tagung der Deutschen Dermatologischen Gesellschaft im Mai 2001 in Berlin, organisierte die Firma Hoffmann-La Roche ein interaktives Fortbildungs-Symposium zum Thema: „Therapie des malignen Melanoms - Wie würden Sie entscheiden?" Eines der Ziele dieses Symposiums war es, die Meinung des Auditoriums zu wichtigen Problemen zum Melanom zu erfahren. Hierzu wurden vor jedem Vortrag der Referenten Stephan Lischner (Kiel), Jens Ulrich (Magdeburg), Matthias Volkenandt (München), Reinhard Dummer (Zürich), Claus Garbe (Tübingen) fünf multiple Choice-Fragen gestellt. Das Auditorium

\section{Abstract}

The discussion about the optimal care of patients with melanoma of the skin is controversial. Five multiple choice questions concerning key questions about the care of melanoma patients were asked to the participants of a symposium and were evaluated by TED. This was done before and after a lecture on this subject. Consequently, the influence of the speaker on the opinion of the lecture audience was evaluated. hatte mittels eines TED-Systems (TED = Kurzwort aus Teledialog, Computer der Stimmabgaben annimmt und hochrechnet) die Möglichkeit eine oder mehrere Antworten auszuwählen. Im Anschluss daran wurden die entsprechenden Vorträge gehalten. Am Ende der Veranstaltung wurden von jedem Referenten jeweils zwei der fünf Fragen wiederholt, um zu sehen, wie und ob die Präsentationen der Referenten die Meinung des Auditoriums beeinflusst haben.

Mittels TED-Umfrage wurde zunächst die Zusammensetzung des 160-köpfigen abstimmenden Auditoriums ermittelt. Danach setzte sich die Zuhörerschaft zusammen aus 20\% niedergelasse-

Institutsangaben

${ }^{1}$ Dermatologische Klinik, Universitätsspital Zürich, Schweiz

${ }^{2}$ Medizinische Fakultät, Universitätsklinik für Dermatologie und Venerologie,

Otto-von-Guericke-Universität Magdeburg

${ }^{3}$ Universitäts-Hautklinik Kiel

${ }^{4}$ Dermatologische Klinik und Poliklinik der Ludwig-Maximilians-Universität, München

${ }^{5}$ Universitäts-Hautklinik, Sektion Dermatologische Onkologie, Eberhard-Karls-Universität, Tübingen

Anmerkung

R. Dummer und A. Hauschild sind Vorsitzende des „interaktiven Melanomsymposiums: Operative und systemische Therapie, Nachsorge - Wie würden Sie entscheiden?“ Unterstützt von Hoffmann-LaRoche Pharma, Satelliten-Symposium der DDG (Mai 2001)

Korrespondenzadresse

Prof. Dr. R. Dummer · Dermatologische Klinik Universitätsspital Zürich · Gloriastraße 31 · 8091 Zürich · Schweiz

E-mail: reinhard.dummer@der.usz.ch

Bibliografie

Akt Dermatol 2002; 28: 456-458 C Georg Thieme Verlag Stuttgart · New York · ISSN 0340-2541 
nen Dermatologen, 4\% Chefärzten, ca. 20\% Oberärzten an Dermatologischen Kliniken, ca. 30\% Assistenzärzten sowie 10\% Ärzten im Praktikum. Die übrigen Teilnehmer gehörten überwiegend der Pharmaindustrie an.

\section{Sicherheitsabstände bei der operativen Versorgung des malignen Melanoms}

Im ersten Vortrag des Symposiums beschäftigte sich Dr. St. Lischner (Kiel) mit der immer noch aktuellen Frage des geeigneten Sicherheitsabstandes bei der Operation von Melanom-Primärtumoren. Die Meinung des Auditoriums vor dem Referat zeigte deutlich, dass das von der DDG empfohlene Stufenschema mit Sicherheitsabständen von 1,2 bzw. $3 \mathrm{~cm}$ bei Tumordicken von bis zu $1 \mathrm{~mm}, 1$ bis $4 \mathrm{~mm}$ bzw. mehr als $4 \mathrm{~mm}$ Tumordicke allgemein eine hohe Akzeptanz findet. Lischner wies jedoch in seinem Vortrag darauf hin, dass bei kritischer Prüfung der Literatur lediglich ein Sicherheitsabstand von $1 \mathrm{~cm}$ bei Tumoren bis $2 \mathrm{~mm}$ Tumordicke bzw. $2 \mathrm{~cm}$ Sicherheitsabstand bei Tumoren bis $4 \mathrm{~mm}$ Dicke als sicher gelten kann. Bei mehr als $4 \mathrm{~mm}$ Tumordicke würde eine noch nicht publizierte, prospektiv-randomisierte Studie mit einer großen Fallzahl in England daraufhin hindeuten, dass auch hier ein kleinerer Sicherheitsabstand ausreichend sein kann. Weder die Gesamtüberlebenszeit noch die Rate der so genannten Lokalrezidive (Satelliten-, Intransit-Metastasen) werde durch einen geringeren Sicherheitsabstand signifikant verschlechtert. In anderen Ländern seien aus diesem Grunde die empfohlenen Sicherheitsabstände bei der Operation von Melanom-Primärtumoren bereits auf 1 bis $2 \mathrm{~cm}$ reduziert worden. Die Daten des Vortrages von Herrn Lischner überzeugten das Auditorium ganz offensichtlich: $30 \%$ der Kollegen, die vorher das DDG-empfohlene $1 / 2 / 3 \mathrm{~cm}$ Stufenschema als Mittel der Wahl ansahen, änderten nach dem Vortrag ihre Meinung. 20\% bevorzugten danach einen Sicherheitsabstand von 1 bis $2 \mathrm{~cm}$ adaptiert an die Tumordicke, 10\% sogar einen maximalen Sicherheitsabstand von $1 \mathrm{~cm}$ bei jeder Tumordicke (Tab.1) [7].

Tab. 1 Prospektiv-randomisierte Studien zum Sicherheitsabstand legen nahe

\begin{tabular}{lcc}
\hline & vor dem Referat & nach dem Referat \\
\hline $\begin{array}{l}\text { ein risikoadaptiertes } \\
\text { Stufenschema: } 1 / 2 / 3 \mathrm{~cm}\end{array}$ & $70 \%$ & $40 \%$ \\
\hline $\begin{array}{l}\text { ein risikoadaptiertes } \\
\text { Stufenschema } 1 / 2 \mathrm{~cm}\end{array}$ & $29 \%$ & $50 \%$ \\
$\begin{array}{l}\text { ein maximaler } \\
\text { Sicherheitsabstand von } 1 \mathrm{~cm}\end{array}$ & $1 \%$ & $10 \%$ \\
\hline
\end{tabular}

\section{Rolle der Sentinel-Lymphknoten-Biopsie}

Herr Jens Ulrich aus Magdeburg behandelte in seinem Referat die operative Versorgung der lokoregionalen Lymphknoten und die Sentinel-Node-Biopsie. Eine der von ihm anfangs gestellten Fragen zeigte, dass mehr als $80 \%$ der Teilnehmer die elektive Lymphknoten-Dissektion für nicht mehr indiziert hielten [4]. Die große prognostische Bedeutung des Sentinel-Lymphknotens wurde im Rahmen dieses Referates sorgfältig herausgearbeitet. Dies führte zu einer Verdoppelung des Anteils der Kollegen (20\% auf $40 \%$ ), die den Status des Sentinel-Lymphknotens als den wichtigsten prognostischen Faktor beim malignen Melanom einordneten [1,11]. Dementsprechend maß das Auditorium der vertikalen Tumordicke nach Breslow, als bislang wichtigstem prognostischen Parameter, nicht mehr eine solch hohe Bedeutung bei (77\% vor dem Referat versus 55\% nach dem Referat). Bezüglich der Frage der histologischen Diagnostik des SentinelLymphknotens bewerteten etwa $20 \%$ der Zuhörerschaft eine Schnellschnittdiagnostik am Kryomaterial zunächst als günstig. Durch die ausführliche Darstellung der Probleme bei der histologischen Aufarbeitung und des Stellenwertes der immunhistochemischen Diagnostik des Sentinel-Lymphknotens waren nach dem Referat nur noch $5 \%$ für eine Schnellschnittdiagnostik. Die kritische Diskussion der Ergebnisse der RT-(Reverse Transkriptase)PCR-Diagnostik des Sentinel-Lymphknotens führte zu einem Rückgang derjenigen (32 auf 25\%), die einen Einsatz dieser molekularbiologischen Methode als Standard ansahen, da sie sensitiver als die Histologie sei [1].

\section{Adjuvante Therapie}

Herr M. Volkenandt diskutierte in seinem Referat den Stand der adjuvanten Therapie des malignen Melanoms [5]. Zunächst ging es um die Frage, für welche Patienten ein Prognosevorteil durch eine Interferon-alpha-Therapie in randomisierten Studien gezeigt wurde. Hier erreichte der Vortrag von Herrn Volkenandt, dass die richtige Antwort (Tumordicke $>1,5 \mathrm{~mm}$ ) und Patienten mit regionären Metastasen von zunächst 75 auf $90 \%$ des Auditoriums anstieg. Im Referat von M. Volkenandt wurde auch die Problematik der optimalen Therapiedauer angesprochen. Die kritische Bewertung der zur Zeit vorliegenden Studien im Rahmen der Präsentation zeigte, dass hier noch ein substanzielles Wissensdefizit besteht. Einige Ergebnisse weisen darauf hin, dass eine adjuvante Therapie mit Interferonen länger als die bisher empfohlenen 12 bis 18 Monaten dauern sollte $[6,10,12]$. Es ist durchaus möglich, dass eine verlängerte Behandlungsdauer zu verbesserten Ergebnissen führt. Die Argumentation von Herrn M. Volkenandt hierzu wurde offensichtlich als stichhaltig bewertet, denn während zunächst nur $40 \%$ des Auditoriums der Meinung waren, dass eine Interferon-Therapie bis zur Tumorprogression sinnvoll sei, waren es nach dem Vortrag von M. Volkenandt $60 \%$.

\section{Palliative Therapie beim metastasierenden Melanom}

Im Rahmen des Vortrages von R. Dummer (Zürich) wurde die aktuelle Datenlage zur Effizienz der medikamentösen Therapie im Stadium IV (Vorliegen von Fernmetastasen) diskutiert. Hier stellt sich die kritische Frage, was für dieses Patientenkollektiv als Standardbehandlung anzusehen ist. Die Benennung einer Standardtherapie fällt in diesem Zusammenhang besonders schwer, da keine Ergebnisse von prospektiven plazebokontrollierten Studien vorliegen, deren Durchführung bei diesem Patientenkollektiv auch aus ethischen Gründen schwierig wäre [3]. Bei der Frage nach einer Standardbehandlung im Stadium IV (Tab. 2) wurde zunächst die Antwort DTIC und Interferon von fast $90 \%$ des Audi- 
Tab. 2 Die Standardtherapie im Stadium IV bei mehreren Metastasen in Lunge, Haut und Lymphknoten ohne weiteren Organbefall ist

\begin{tabular}{lcc}
\hline & vor dem Referat & nach dem Referat \\
\hline Dendritic Cell-Vakzinierung & $2 \%$ & $0 \%$ \\
DTIC alleine & $31 \%$ & $39 \%$ \\
Interleukin-2 hochdosiert alleine & $2 \%$ & $2 \%$ \\
Interferon- $\alpha$ & $5 \%$ & $2 \%$ \\
\hline DTIC + Interferon & $87 \%$ & $63 \%$ \\
\hline
\end{tabular}

toriums gewählt [9] und nur von 30\% für die DTIC alleine (Doppelantworten möglich). Nach der Präsentation entschieden sich doch 39\% der Zuhörerschaft für eine DTIC-Monotherapie, während nur noch $63 \%$ die Option DTIC plus Interferon wählten (Tab. 2). Die kritische Darstellung der aktuell publizierten Studien zur Wirksamkeit von systemischen Therapien, v. a. in Bezug auf die Gesamtüberlebenszeit, führte auch zu einer deutlich pessimistischeren Einschätzung des Nutzens der aktuell verfügbaren Therapieoptionen [3]. Während vor Beginn des Referates $53 \%$ des Auditoriums der Meinung waren, dass mehr als $30 \%$ aller Patienten mit Melanommetastasen von einer Behandlung profitieren, waren es nach dem Referat nur noch 30\%. Aufgrund dieser Datenlage empfiehlt sich primär die Behandlung im Rahmen großer Multizenterstudien.

\section{Nachsorge}

Im letzten Referat diskutierte C. Garbe (Tübingen) kritisch die Nachsorge von Melanompatienten, wobei er die Effizienz der klinischen (körperlichen) Untersuchungen und die verschiedener bildgebender Verfahren, wie Röntgen-Thorax, Sonographie des Abdomens und der Lymphknoten sowie ComputertomographieUntersuchungen, bewertete. Herr C. Garbe wies auf die Bedeutung einer sorgfältigen körperlichen Untersuchung hin. Die klare Darstellung der Tübinger Ergebnisse zur Bedeutung der Sensitivität der Lymphknoten-Sonographie bei der Entdeckung von Lymphknoten-Metastasen [2] wurde reflektiert durch einen hohen Prozentsatz der Zuhörerschaft, die die LymphknotenSonographie als wichtigstes bildgebendes Verfahren einschätzten $(68 \%)$. Die Frage von Herrn Garbe nach dem wichtigsten Laborparameter in der Nachsorge wurde vom Auditorium überraschenderweise so beantwortet, dass $60 \%$ das lösliche S-100B-Molekül im Serum für den wichtigsten Parameter halten. Dieses Ergebnis reflektiert die Hauptaussagen aktueller Publikationen $[8,13]$, während weniger als $40 \%$ die Bestimmung der Laktatdehydrogenase am wichtigsten einschätzen. Dies zeigt die hohe Bereitschaft des Auditoriums sich fortzubilden. Die aktuellen Publikationen, die eine mögliche Rolle für die Bestimmung des löslichen S-100B Proteins in der Nachsorge zeigen, sind erst in den letzten Jahren erschienen.

\section{Schlussfolgerung}

Die TED-Befragung des Publikums im Rahmen des Symposiums erreichte eine bessere Einbindung des Auditoriums in die Veranstaltung. Diese Technik sollte in Zukunft vermehrt eingesetzt werden. Die Befragung der sicher nicht repräsentativen Zuhörerschaft zeigt, dass der Wissensstand der anwesenden Dermatologen zum malignen Melanom weitgehend als gut zu bewerten ist. Es bestehen jedoch einige festgefügte Meinungen zu kritischen Punkten wie Sicherheitsabstand, Wirksamkeit der systemischen Therapie im Stadium IV und Nachsorge, die sich aufgrund der vorliegenden Datenlage unter besonderer Berücksichtigung kontrollierter Studien entsprechend den Kriterien der Evidence based medicine nicht mehr sicher halten lassen. Im Sinne einer rationellen medizinischen Versorgung von Melanompatienten in Zeiten knapper Ressourcen müssen diese Punkte weiter bearbeitet und untersucht werden, um so weiterhin eine wissenschaftlich gut fundierte Betreuung der Melanompatienten gewährleisten zu können.

\section{Literatur}

${ }^{1}$ Blaheta HJ, Paul T, Sotlar K et al. Detection of melanoma cells in sentinel lymph nodes, bone marrow and peripheral blood by a reverse transcription-polymerase chain reaction assay in patients with primary cutaneous melanoma: association with Breslow's tumour thickness. Br J Dermatol 2001; 145: 195-202

2 Blum A, Schlagenhauff B, Stroebel W et al. Ultrasound examination of regional lymph nodes significantly improves early detection of locoregional metastases during the follow-up of patients with cutaneous melanoma: results of a prospective study of 1288 patients. Cancer 2000; 88: 2534-2539

${ }^{3}$ Crosby T, Fish R, Coles B, Mason MD. Systemic treatments for metastatic cutaneous melanoma (Cochrane Review). The Cochrane Library, Update Software Oxford: 2000

${ }^{4}$ Dummer R, Bösch U, Panizzon R et al. Guideline zur Therapie und Nachsorge des kutanen Melanoms. Schw. Aerztezeitung 2001; 82: $1081-1086$

${ }^{5}$ Dummer R, Nestle FO, Burg G. Immuntherapie des Melanoms. Dtsch med Wschr 2000; 125: 1240-1242

${ }^{6}$ Grob JJ, Dreno B, delaSalmoniere P et al. Randomised trial of interferon alpha-2a as adjuvant therapy in resected primary melanoma thicker than $1.5 \mathrm{~mm}$ without clinically detectable node metastases. Lancet 1998; 351(9120): $1905-1910$

${ }^{7}$ Hauschild A, Eiling S, Lischner S et al. Sicherheitsabstände bei der Exzision des primären Melanoms: Diskussionsvorschläge aufgrund von Ergebnissen aus kontrollierten klinischen Studien. Hautarzt 2001; 52 (11): $1003-1010$

${ }^{8}$ Hauschild A, Engel G, Brenner W et al. Predictive value of serum S100B for monitoring patients with metastatic melanoma during chemotherapy and/or immunotherapy. Br J Dermatol 1999; 140: 1065-1071

${ }^{9}$ Huncharek M, Caubet JF, McGarry R. Single-agent DTIC versus combination chemotherapy with or without immunotherapy in metastatic melanoma: a meta-analysis of 3273 patients from 20 randomized trials. Melanoma Res 2001; 11: 75-81

${ }^{10}$ Kirkwood JM, Strawderman MH, Ernstoff MS et al. Interferon alfa-2b adjuvant therapy of high-risk resected cutaneous melanoma: the Eastern Cooperative Oncology Group Trial EST 1684. J Clin Oncol 1996; 14: $7-17$

${ }^{11}$ Morton DL, Wen DR, Wong JH et al. Technical details of intraoperative lymphatic mapping for early stage melanoma. Arch Surg 1992; 127: $392-399$

12 Pehamberger H, Soyer HP, Steiner A et al. Adjuvant interferon alfa-2a treatment in resected primary stage II cutaneous melanoma. Austrian Malignant Melanoma Cooperative Group. J Clin Oncol 1998; 16: $1425-1429$

${ }^{13}$ Schlagenhauff B, Schittek B, Ellwanger U et al. Significance of serum protein $\mathrm{S} 100$ levels in screening for melanoma metastasis: does protein S100 enable early detection of melanoma recurrence? Melanoma Res 2000; 10: $451-459$ 